\title{
RESEARCH
}

\section{THE EFFECT OF FEAR OF COVID-19 AND SOCIAL ISOLATION ON THE FRAGILITY IN THE ELDERLY}

Turkish Journal of Geriatrics

DOI: 10.31086/tjgeri.2021.196

2021; 24(1): 23-31

\section{- Burak METE ${ }^{1}$}

- Ferdi TANIR ${ }^{1}$

- Ceren KANAT ${ }^{1}$

CORRESPONDANCE

\section{${ }^{1}$ Burak METE}

Cukurova University, Public Health Department, Adana, Turkey

\section{Phone: +905447113164}

e-mail: burakmete2008@gmail.com

Received: Nov 14, 2020

Accepted: Mar 01, 2021

${ }^{1}$ Cukurova University, Public Health

Department, Adana, Turkey

\section{Abstract}

Introduction: The most important matter on which a consensus is built about COVID-19 disease is that the elderly are one of the most vulnerable risk groups. In this study, we aimed to evaluate the impact of the COVID-19 pandemic on the fragility of the elderly.

Materials and Methods: The study is a cross-sectional study conducted at the end of the sixth month of the pandemic. Our study included 319 elderly individuals. The elderly individuals were reached at the primary level health centers. "FRAIL Frailty Scale" and "Coronavirus Fear Scale" were used in the evaluation.

Results: The results showed that $72.4 \%$ of the elderly population could not comply with the routine health checks and chronic complaints increased to $39.3 \%$ during social isolation. In the first six-month period of the pandemic, it was found that the prefrail and fragile elderly population increase by $4.7 \%$ and $6.6 \%$, respectively. The fragility risk increased by 1.03 times (OR:1.001-1.007) as COVID-19 fear increased, while 2.2 times (OR:1.23-3.94) in those with diabetes, 1.88 times (OR:1.01-3.49) in those with cardiovascular diseases, and 2.15 times (OR:1.07-4.34) in those who postponed their routine health checks.

Conclusion: Early stage results of social isolation measures taken for the elderly within the scope of the pandemic indicated that fear of coronavirus infection increased fragility among the elderly. Elderly health evaluations should continue in the further stages of the pandemic.

Keywords: Pandemics; Frail Elderly; Social Isolation 


\section{INTRODUCTION}

COVID-19, which broke out in November 2019 in Hubei City of China, caused by SARS-CoV-2 virus, has influenced the entire world (1). While 108.5 million people have been infected so far, 2.38 million people have died because of the infection (2). It is estimated that the fatality rate of the novel coronavirus disease is around $2-3 \%$. According to the data of the "National Medical Commission" of China, $80 \%$ of the casualties are over 60 years of age and $75 \%$ has pre-existing medical issues (cardiovascular disease, diabetes, asthma, etc.). It is seen that the case fatality rate among the elderly is higher than the young, with $24.6 \%$ in the $65-74$ age group and $47.7 \%$ in the over 75 years of age group (3). When the case fatality rates in elderly cases in Turkey are analyzed, a similar picture is observed: the fatality rate is $19.2 \%$ in men and $10.1 \%$ in women between 65 and 79 years of age; while it is $37.7 \%$ and $25.8 \%$ in men and women, respectively, among people aged 80 years and older (4). The most important matter on which a consensus is built about COVID-19 disease is that the elderly are one of the most vulnerable risk groups. In view of this, public authorities implement measures that address the needs of the elderly and try to increase their compliance through these measures. The elderly are expected to be more inclined to be isolated if need be and they comply with the preventive measures more easily than the young (5). Although social isolation seems beneficial in preventing the spread of the disease in elderly individuals, prolonging this process might have adverse psychological and physiological consequences. Fragility is a medical syndrome that increases dependency on another individual in daily life and/or death risk and is characterized by reduced physical function, resistance, and strength involving several factors and causes. Fragility in the elderly is regarded as a significant cause of morbidity and mortality. Although fragility has several adverse outcomes, it is a condition that can be prevented and remedied (6). We anticipate that prolonging social isolation, fear of getting sick, and postponing medical services will increase fragility among the elderly during the pandemic. The aim of this study was to evaluate how uninterrupted social isolation affected elderly individuals and how fear of coronavirus affected fragility levels at the sixth months of pandemics.

\section{MATERIALS AND METHODS}

This descriptive study was conducted in Adana Province, Turkey, in October 2020 (at the end of the sixth month of the pandemic). Approvals for the study obtained from the Turkish Ministry of Health and Çukurova University's Ethical Committee (decree number: 104). The study population consisted of people aged 65 years and older. According to the results of the pilot study conducted by taking $80 \%$ as power and $95 \%$ confidence interval as reference, the minimum number for the sample size for the analysis was calculated as 247 ( $\mathrm{r} 0=0.184$, $r 1=0.350$ ). A total of 319 people were included in the study. Convenience sampling was used as the sampling method. The elderly individuals were reached at the primary level health centers (i.e. family medicine centers, community health centers) of Çukurova University, Faculty of Medicine, Public Health Department's Practice and Research Areas in Adana city. Questionnaire forms were filled by face-to-face interviews. Informed consent was obtained from participants. While filling out the questionnaires, infection measures were taken into consideration like physical distancing, wearing of masks, and hand hygiene. The data collection form consisted of four parts: sociodemographic data, Mini Nutritional Assessment scale, FRAIL scale, and Fear of Coronavirus Scale. The socio-demographic form included questions about age, sex, place of residence, education, income, occupation, height, weight, presence of chronic diseases, smoking habit, number of medicines taken daily, number of people in the household, and process of adaptation to social isolation. 


\section{Mini Nutritional Assessment Scale Short Form (MNA-SF)}

The validity and reliability of the MNA scale in our country was made by Sarıkaya D. in 2013. MNA-SF contains six items that show a high correlation with conventional nutritional assessment. In MNA-SF, scoring is done according to a change in the patient's appetite, any weight loss in the last 3 months, patient mobility, presence of psychological distress or acute disease in the last 3 months, presence of neuropsychological problems, and the patient's body mass index. When MNA-SF is used alone, the results are classified as adequate nutrition (11-14), at risk (7-11), and malnutrition (<7) (7).

\section{Fear of COVID-19 Scale}

This scale consists of a single dimension and comprises 7 items. The scale does not contain any reverse item. The total score obtained from all the scale items reflects the level of Coronavirus (COVID-19) fear that the individual experiences. The scores that can be obtained from the scale varies between 7 and 35. Higher scores obtained from the scale indicate a high level of Coronavirus fear (8). The validity and reliability of the scale were assessed by Bakioğlu et al. (9).

\section{FRAIL Scale}

The FRAIL scale was used to determine a patient's fragility state. A validity-reliability study of the Turkish FRAIL scale was conducted in 2017 by Muradi et al. This scale has 5 components: fatigue, resistance, ambulation, illness, and loss of weight. Each component is scored as 0 or 1 . The total score varies between 0 and 5 . Scores are evaluated as follows: 0 as normal; $1-2$ as prefrail and $3-5$ as frail (10). Fragility is evaluated in two sub-dimensions: the pre-pandemic period and the pandemic period (the end of the sixth month). The individuals were asked to evaluate the sub-dimensions before and at the sixth month of the pandemic.

\section{Evaluation of the change in the fragility}

The participants were asked to evaluate the sub-dimensions of the frailty scale once in the pre-pandemic period and later again at the end of sixth month after the start of the pandemic and the declaration of curfews (social isolation).

Fatigue: "Before the start of the pandemic, how much of the time during the past 4 weeks did you feel tired?" "After the start of the pandemic (now), how much of the time during the past 4 weeks did you feel tired?"

Resistance: "Before the start of the pandemic, by yourself and not using aids, did you have any difficulty walking up 10 steps without resting?" "After the start of the pandemic (now), by yourself and not using aids, do you have any difficulty walking up 10 steps without resting?

Ambulation: "Before the start of the pandemic, by yourself and not using aids, did you have any difficulty walking several hundreds of meters?" "After the start of the pandemic (now), by yourself and not using aids, do you have any difficulty walking several hundreds of meters?"

Illnesses: "Before the start of the pandemic, how many chronic ilnesses did you have?" "After the start of the pandemic (now), how many chronic ilnesses do you have?"

Loss of weight: "Before the start of the pandemic, how much did you weigh with your clothes on but without shoes?" "After the start of the pandemic (now), how much do you weigh with your clothes on but without shoes?" Percent change $>5$ in weight was interpreted as frailty.

Total frailty scale score was calculated by summing the scores for each sub-dimension for both the pre-pandemic period and at the sixth month of the pandemic. The mathematical difference by subtracting the pre-pandemic period scores from the post-pandemic scores yielded the final score change, with positive scores interpreted as in- 
crease, negative scores as decrease and zero as unchanged.

\section{Evaluation of the social isolation}

The compliance of elderly people during social isolation periods (curfews) and the changes that occurred during this period were asked (adapting to social isolation measures, exercising at home during the social isolation periods, attending routine health checks, falling at home, increase in chronic complaints, psychological distress, change in eating habits, onset or increase of amnesia).

\section{Statistical Analysis}

SPSS 22 software was used for the data analysis. Normal distribution was tested by the KolmogorovSmirnov test. Marginal homogeneity test, paired t-test, Wilcoxon test, and Binary logistic regression analysis were performed to analyse the data. Binary logistic regression analysis was performed to estimate the change in fragility and to evaluate the effect of independent variables on fragility risk. In regression analyzes, the dependent variable was the change in fragility. The reference category referred to the participants scored "normal" both pre-pandemic period and at sixth month of the pandemics. The risk category consisted of the participants that had scored "normal" before the pandemic, but switched to "prefragile" or "fragile" at the sixth month of the pandemics, i.e. the reference was "normal" score and the risk parameter was "prefragile" or "fragile". In effect size analyses, Cohen's $d$ value $(d) \geq 1$ indicated a very large effect, 0.8 a big effect, 0.5 a moderate effect, and 0.2 a small effect. $p<0.05$ was considered significant.

\section{RESULTS}

The average age of the 319 elderly individuals who participated in our study was $71.66 \pm 6.17$ (range: 65-95 years). The sociodemographic characteris- tics of the study population are presented in Table 1. In the study population, $88.1 \%$ of the individuals reported presence of chronic diseases, with hypertension as the most frequent one. Malnutrition was found to be $6.3 \%$ in elderly individuals (Table 1 ).

When the change in frailty among elderly individuals between the pre-pandemic period and at the end of the first six months of the pandemic was analyzed, it was found that the number of elderly individuals in the "normal scored" group decreased by $11.4 \%$, and consequently the increase in rate was $4.7 \%$ in the prefrail group and $6.6 \%$ in the frail group $(p<0.001)$ (Table 2). The change in frailty was found not to differ significantly in relation to sex, age, income, malnutrition and smoking groups was analyzed, no significant difference was found $(p<0.05)$.

The difference between pre-pandemic and pandemic fragility scores was found to be statistically significant $(p<0.001)$. For the first six months of the pandemic, the effect of the pandemic on the fragility scores was found to be small with Cohen's $d$ of 0.222 (Table 2).

The logistic regression model set was found to be significant (Omnibus test $p=0.006$ ) for predicting the changes in the frailty group, including the presence of chronic diseases and the coronavirus fear score of the participants. The accuracy of the model was found to be $73.8 \%$ with Nagelkerke R square value of 0.094 . The most contributing variable was determined as "having diabetes" with an R square value of 0.040 . Practices like social isolation or curfews, intending to protect the elderly, were found to increase the risk of switching from the normal to the prefrail/frailty group at the sixth month of the pandemic by 2.2 times for DM patients and 1.88 times for those with cardiovascular disease. The increase in the scores of the coronavirus fear scale increased the risk of switching fom the normal to the prefrail/ frailty by 1.03 times (Table 3 ).

The adaptation of elderly people to the process of curfews and the changes they experienced during this period are presented in Table 4. It was 
Table 1. Sociodemographic characteristics and medical conditions of the individuals

\begin{tabular}{|l|c|}
\hline Characteristics & $\mathrm{n}(\%)$ \\
\hline Sex (Male/female) & $156(48.9) / 163(51.1)$ \\
\hline Age (65-74/75-84/85 and above) & $231(72.4) / 75(23.5) / 13(4.1)$ \\
\hline Education (illiterate/primary/elementary/high school/university) & $103(32.3) / 147(46.1) / 47(14.7) / 22(6.9)$ \\
\hline Income (2500 and lower/2501-5000/5001-7500/7501 TL and higher) & $126(39.5) / 50(15.7) / 66(20.7) / 71(22.3)$ \\
\hline Occupation (housewife/worker/officer/tradesmen-farmer) & $24(7.5) / 169(53.0) / 126(39.5)$ \\
\hline Current occupation (working/not working/housewife) & $280(88.1) / 38(11.9)$ \\
\hline Chronic diseases (yes/no) & $187(66.5)$ \\
\hline Hypertension (HT) & $146(52.0)$ \\
Diabetes mellitus (DM) & $104(37.1)$ \\
Cardiovascular diseases (CVD) & $62(22.1)$ \\
COPD & $43(15.3)$ \\
Rheumatological diseases & $10(3.6)$ \\
Malignancy & $14(5.1)$ \\
Other & (191(60.3) / 106(3.4)/ 20 (6.3) \\
\hline Malnutrition (normal / at risk / malnutrition) & $62(19.5) / 207(65.1) / 49(15.4)$ \\
\hline Smoking (yes / no / quit) & $37(11.8) / 128(40.8) / 123(39.2) / 26(8.3)$ \\
\hline Number of daily drug (0/1-3/4-7/8 and above) & \\
\hline
\end{tabular}

Table 2. Change in fragilty before and after the pandemic

\begin{tabular}{|c|c|c|c|c|}
\hline Fragility group & $\begin{array}{c}\text { Before } \\
\text { the pandemic } \\
\mathrm{n}(\%)\end{array}$ & $\begin{array}{c}\text { At } 6^{\text {th }} \text { month } \\
\text { of the pandemic } \\
n(\%)\end{array}$ & $\begin{array}{c}\text { Change } \\
(\%)\end{array}$ & $p$ \\
\hline Normal & $137(43.4)$ & $101(32.0)$ & -11.4 & \\
\hline Prefrail & $125(39.6)$ & $140(44.3)$ & 4.7 & $<0.001$ \\
\hline \multirow[t]{2}{*}{ Fragile } & $54(17.1)$ & $75(23.7)$ & 6.6 & \\
\hline & X \pm S.D. & X \pm S.D. & Cohen's d & $\mathrm{p}$ \\
\hline Fragility score & $1.16 \pm 1.23$ & $1.44 \pm 1.29$ & 0.222 & $<0.001$ \\
\hline
\end{tabular}

Table 3. Logistic regression model for predicting the impact of pre-existing health problems and fear of Covid-19 on fragilty

\begin{tabular}{|l|c|c|c|c|c|}
\hline \multirow{2}{*}{\multicolumn{1}{c|}{ Variables }} & & & & \multicolumn{2}{c|}{ 95\% C.I.for O.R. } \\
\cline { 2 - 6 } & B & $\mathbf{p}$ & O.R. & Lower Upper \\
\hline Fear of COVID-19 & 0.035 & $\mathbf{0 . 0 4 2}$ & 1.036 & 1.001 & 1.072 \\
\hline HT & 0.430 & 0.176 & 1.537 & 0.824 & 2.867 \\
\hline DM & 0.791 & $\mathbf{0 . 0 0 7}$ & 2.206 & 1.235 & 3.940 \\
\hline COPD & -0.045 & 0.898 & 0.956 & 0.476 & 1.917 \\
\hline Malignancy & 0.295 & 0.686 & 1.343 & 0.322 & 5.601 \\
\hline CVD & 0.634 & $\mathbf{0 . 0 4 4}$ & 1.885 & 1.016 & 3.499 \\
\hline Constant & -2.229 & $<0.001$ & 0.108 & & \\
\hline
\end{tabular}


found that $77.1 \%$ of the participants were able to adapt to this process, $78.4 \%$ were physically inactive during this period as they were unable to exercise at home, $72.4 \%$ could not attend routine health checks, $60.7 \%$ experienced increase in their pre-existing complaints, $73.6 \%$ had psychological difficulties, $34.6 \%$ had a change in eating habits, and $17.9 \%$ experienced onset of amnesia or increase in it during curfews (Table 4).

Another logistic regression model set was found to be significant for predicting the effect of the adaptation of elderly people to the process of curfews and the changes they experienced during this period on the frailty scores (Omnibus test $p<0.001$ ). The independent variables of the model were exercis- ing during the curfews, being able to attend routine health checks, change in eating habits, falling at home, change in existing complaints and onset of amnesia. The accuracy of the model was found to be $74.9 \%$ with Nagelkerke R square value of 0.118 . Practices like social isolation or curfews, intending to protect the elderly, were found to increase the risk of switching from the normal to the prefrail/frailty group at the sixth month of the pandemic by 2.15 times for participants who could not attend their routine health checks because of the curfews and social isolation measures or postponed because of the fear of getting sick, 1.85 times for the elderly who changed their eating habits, and 1.97 times for the elderly with an increase in their pre-existing complaints (Table 5).

Table 4. Adaptation of elderly individuals to social isolation process

\begin{tabular}{|c|c|c|}
\hline & $\begin{array}{c}\text { Yes } \\
n(\%)\end{array}$ & $\begin{array}{c}\text { No } \\
\text { n (\%) }\end{array}$ \\
\hline Adaptation to social isolation measures & $246(77.1)$ & $73(22.9)$ \\
\hline Exercising at home during social isolation & $69(21.6)$ & $250(78.4)$ \\
\hline Compliance with routine health checks during social isolation & $88(27.6)$ & $231(72.4)$ \\
\hline History of fall at home during social isolation & $44(13.8)$ & $274(86.2)$ \\
\hline Increased chronic complaints during social isolation & $125(39.3)$ & $193(60.7)$ \\
\hline Having psychological difficulty during social isolation & $234(73.6)$ & $84(26.4)$ \\
\hline Change in nutritional habits during social isolation & $111(34.9)$ & $207(65.1)$ \\
\hline Onset of or increase in amnesia during social isolation & $57(17.9)$ & $261(82.1)$ \\
\hline
\end{tabular}

Table 5. Logistic regression model for predicting the impact of adaptation to social isolation process on frailty

\begin{tabular}{|c|c|c|c|c|c|}
\hline \multirow{2}{*}{ Variables } & \multirow[b]{2}{*}{ B } & \multirow[b]{2}{*}{$p$} & \multirow[b]{2}{*}{ O.R. } & \multicolumn{2}{|c|}{ 95\% C.I.for O.R. } \\
\hline & & & & Lower & Upper \\
\hline Exercise & 0.495 & 0.186 & 1.640 & 0.788 & 3.415 \\
\hline Health check & 0.769 & 0.031 & 2.157 & 1.072 & 4.340 \\
\hline Fall at home & 0.294 & 0.441 & 1.342 & 0.635 & 2.834 \\
\hline Complaints & 0.680 & 0.021 & 1.974 & 1.108 & 3.516 \\
\hline Nutritional habits & 0.617 & 0.031 & 1.854 & 1.059 & 3.244 \\
\hline Amnesia & 0.046 & 0893 & 1.047 & 0.534 & 2.054 \\
\hline Constant & -1.299 & $<0.001$ & 0.273 & & \\
\hline
\end{tabular}




\section{DISCUSSION}

The COVID-19 pandemic has compelled governments to make solid public health measures to minimize the impact of the disease. These initiatives include imposing curfew for the elderly as well as social distancing. The experts concur that the elderly are the most vulnerable group. In line with this, public authorities implemented measures to prevent infection and increase elderly individuals' compliance with such measures (11). Furthermore, epidemiologists emphasize that the most significant risk factor of mortality due to COVID-19 is age, and people over 65 are at higher risk (12).

This study evaluated the impact of the measures taken to reduce the spread of the disease among the elderly in the first 6 months of the pandemic on the fragility. It was found that $11.4 \%$ of the people who were in the normal group before the start of the pandemic switched to the prefrail $(4.7 \%)$ and fragile (6.7\%) groups at the first 6 months. In the first six months, the impact on frailty was small, but increased 1.03 times in parallel with the increase in the fear of coronavirus. When the chronic diseases that could increase the risk of frailty were examined, the risk of switching from the normal to the fragile group of the elderly with DM and CVD was found to be 2.2 and 1.88 times, respectively. In addition, the same risk increase was found to be 2.15 times in the elderly who postponed their health checks due to the pandemic, 1.85 times in those who changed their eating habits, and 1.97 times in the elderly people with increased complaints. As of now, studies on COVID-19 have consistently shown that older age and comorbidity are major risk factors for adverse outcomes and mortality. Not all older adults appear to be equally vulnerable to COVID-19 (12). Frail older adults have an increased vulnerability to such a stressor event; they tend to be more seriously affected by acute disease in general and they often do not regain their baseline level of health and independence, as compared with non-frail older adults of the same age group (13). In our study, it was found that comorbid conditions such as DM and CVD increased the risk of frailty in the social isolation process.

Studies in the literature mostly focused on the clinical consequences of the COVID-19 disease of frailty. Indeed, frailty was only investigated in regards to its association with overall mortality, hospital infections, intensive care units admission rates, and disease phenotypes in the available studies (14). Studies have found that the risk of adverse clinical outcomes such as mortality and going to intensive care increased in frail elderly compared to normal elderly (15). There are not sufficient studies investigating the effect of social isolation measures taken during the pandemic process on elderly frailty. In a review about the effect of COVID-19 on the elderly Ilgili stated that extensive restriction processes such as social isolation taken since the early stages of the pandemic will have significant effects on elderly health (16). These have dimensions bringing isolation and pandemics are not merely biological phenomena they also affect society at a large. In many societies, also depending on the culture and common lifestyle, the elderly people are living alone, and loneliness is identified as a potential risk factor for cognitive disorders and depression (17). Similarly, in our study, the onset of or increase in amnesia were observed in $17.9 \%$ of the elderly during the social isolation process.

According to the literature, psychiatric history, consist of disaster-related trauma or pre-existing mental health problems, also necessitates close attention, and extra support during the pandemic (18). The fear of the pandemic is also suspected to have an increased psychological effect on the aged concerning their pre-existed awareness of their vulnerability. Similarly, in our study, the fear of coronavirus was found to increase frailty. The fear of death and the existential fear of losing the loved ones is also reported. The psychological impact of quarantine is accepted to be wide-ranging, substantial, and can be long-lasting (18). Butler et al. who in- 
vestigated the impact of nutrition on COVID-19 susceptibility and its long-term results, reported that pandemic would affect all age groups, but especially the elderly, increased consumption of diets containing fat and highly-refined carbohydrates would increase the prevalence of obesity and DM, and consequently the risk of severe COVID-19 disease and its mortality would increase (19). In our study, it was found that the eating habits were found to be changed in $34.9 \%$ of the elderly and the risk of frailty to increase 1.8 times in those who had changed their eating habits.

While changes in diet are reported to increase the risk of severe disease by possible inflammatory mechanisms observed in DM or obesity, the results of our study showed that, as the change in diet increased the risk of frailty, could lead to the severe prognosis of the disease. Frailty and multi-morbidity are two related conditions in older adults. Most frail individuals are also multi-morbid, but fewer multi-morbid individuals also present frailty (20). Social isolation measures taken during the pandemic can contribute to this bilateral relationship. Social isolation may cause exacerbation of pre-existing diseases and increase in frailty, and the increase in frailty itself may cause exacerbation of comorbid situations. Our study has findings supporting this:

\section{REFERENCES}

1. Esakandari H, Nabi-Afjadi M, Fakkari-Afjadi J, Farahmandian N, Miresmaeili SM, Bahreini E. A comprehensive review of COVID-19 characteristics. Biol Proced Online 2020;4:22-19. (PMID: 32774178)

2. Worldometer. Covid-19 Coronavirus Pandemıc; 2020. [Internet]. Available from: https://www.worldometers. info/coronavirus/\#ref-21 Accessed: 15.10.2020

3. The Novel Coronavirus Pneumonia Emergency Response Epidemiology Team. The Epidemiological Characteristics of an Outbreak of 2019 Novel Coro- comorbid conditions such as DM and CVD were found to increase the risk of frailty. Increased frailty may not only lead to the worsening in the prognosis of comorbid diseases, but also make elderly people more vulnerable to COVID-19.

Being conducted in a single region and using non-probability sampling are the limitations of the study.

According to the results of this study, it was found that there was an increase in the number of frail elderly individuals at the end of the first six months of the pandemic. Comorbid conditions that increased the risk of frailty were found to be DM and CVD, as well as the fear of coronavirus, the change in eating habits during the social isolation due to the pandemic, and postponing routine health checks increased the risk of frailty. More studies are needed to investigate the effects of the pandemic and the social isolation measures taken on the frailty of the elderly. It may be recommended to conduct studies evaluating the long-term consequences of the pandemic and to make regulations regarding the conditions that could increase frailty in the elderly.

Conflict of Interest: the authors do not declare a conflict of interest.

navirus Diseases (COVID-19). China CDC Weekly 2020;2(8):113-22. (DOI: 10.46234/ccdcw2020.032)

4. Uçar A, Arslan Ş, Manap H, et al. A Interactive Web Based Dashboard for Covid-19 Pandemic Real Time Monitorization In Turkey: TURCOVID19. Anatolian Clinic the Journal of Medical Sciences 2020;25:154155. (in Turkish) (DOI: 10.21673/anadoluklin.726347)

5. Daoust JF. Elderly people and responses to COVID-19 in 27 Countries. PLoS One 2020;2:15(7):e0235590. (PMID: 32614889)

6. Tribess S, de Oliveira RJ. Biological fragility syndrome in the elderly: systematic review. Rev Salud Publica 2011;13(5):853-64. (PMID: 22634952) 
7. Sarikaya D, Halil M, Kuyumcu ME, et al. Mini nutritional assessment test long and short form are valid screening tools in Turkish older adults. Arch Gerontol Geriatr 2015;61(1):56-60. (PMID: 25944059)

8. Ahorsu DK, Lin CY, Imani V, Saffari M, Griffiths MD, Pakpour AH. The Fear of COVID-19 Scale: Development and Initial Validation. Int J Ment Health Addict 2020;27:1-9. (PMID: 32226353)

9. Bakioğlu F, Korkmaz O, Ercan H. Fear of COVID-19 and Positivity: Mediating Role of Intolerance of Uncertainty, Depression, Anxiety, and Stress. Int J Ment Health Addict. 2020;28:1-14. (PMID: 32837421)

10. Morley JE, Malmstrom TK, Miller DK. A simple frailty questionnaire (FRAIL) predicts outcomes in middle aged African Americans. J Nutr Health Aging 2012;16(7):601-8. (PMID: 22836700)

11. 11-Utych SM, Fowler L. Age-based messaging strategies for communication about COVID-19. Journal of Behavioral Public Administration 2020;3(1):1-14. (DOI: 10.30636/jbpa.31.151).

12. 12-Zhou F, Yu T, Du R, et al. Clinical course and risk factors for mortality of adult inpatients with COVID-19 in Wuhan, China: a retrospective cohort study. Lancet 2020;395(10229):1054-62. (PMID: 32171076)

13. Clegg A, Young J, lliffe S, Rikkert MO, Rockwood K. Frailty in elderly people. Lancet. 2013;381(9868):75262.(PMID: 23395245)
14. Nickel CH, Rueegg M, Pargger H, Bingisser R. Age, comorbidity, frailty status: effects on disposition and resource allocation during the COVID-19 pandemic. Swiss Med Wkly. 2020;150:w20269. (PMID: 32352150)

15. Maltese G, Corsonello A, Di Rosa M, et al. Frailty and COVID-19: A Systematic Scoping Review. J Clin Med. 2020;9(7):2106. (PMID: 32635468)

16. Illgili Ö, Kutsal YG. Impact Of Covid-19 Among The Elderly Population. Turkish Journal of Geriatrics 2020;23(4):419-423. (DOI : 10.31086/tjgeri.2020.179)

17. Banerjee D. Age and ageism in COVID-19: Elderly mental health-care vulnerabilities and needs. Asian J Psychiatry. 2020;51:102154. (PMID: 32403024)

18. Brooks SK, Webster RK, Smith LE, et al. The psychological impact of quarantine and how to reduce it: rapid review of the evidence. Lancet. 2020;395:91220. (PMID: 32112714)

19. Butler MJ, Barrientos RM. The impact of nutrition on COVID-19 susceptibility and long-term consequences. Brain Behav Immun. 2020;87:53-54. (PMID: 32311498)

20. Vetrano DL, Palmer K, Marengoni A, et al. Frailty and Multimorbidity: A Systematic Review and Meta-analysis. J Gerontol A Biol Sci Med Sci. 2019;74(5):659-666. (PMID: 29726918). 